\title{
BIBLIOGRAFIA
}

\section{Bibliografía de y sobre Julio Cortázar}

\section{INTRODUCCIÓN}

Julio Cortázar es un escritor que ha suscitado grandes polémicas que oscilan entre ataques violentos, personales e injustificados (podemos citar a I. Anzoátegui y Manuel Pedro González como ejemplos típicos de esta tendencia), y elogios de desbordante entusiasmo de críticos (como Fernández Retamar) que proclaman que Rayuela es tan importante para la literatura hispanoamericana como el Ulysses de Joyce para los escritores de lengua inglesa. Al respecto, Cortázar dice: "la crítica es como Periquita y hace lo que puede" (Ultimo round, p.b. 29), pero confiesa que los descubrimientos de algunos críticos lo perturban, y también lo dejan "entre perplejo y esperanzado" (31).

Dejando todo juicio de valores de lado, es indudable que Cortázar incita a la crítica, $y$ en este sentido ha contribuido tanto a esta disciplina como a la ficción, haciendo que el crítico, al igual que el lector, se convirtiera en "camarada de camino."

La labor crítica sobre el escritor es numerosa y constante. Si hacemós un gráfico, podemos ver que a pesar de que en 1949 aparece el primer articulo crítico sobre la obra del escritor, hay un lapso de ocho años hasta que aparecen otros. Desde 1957 hasta 1962 hay un promedio de 1 ó 2 por año. Luego, en 1963, coincidiendo con la aparición de $R a-$ yuela y el subsiguiente "descubrimiento" de su autor, el número de escritos criticos va en aumento hasta que alcanza su apogeo entre los' años 1964 y 1971, destacándose 1967, 1968 y 1969 con la más elevada cantidad. También, por otra parte las tesis doctorales sobre el escritor van en constante aumento desde 1969, demostrando el enorme entusiasmo que ha suscitado entre los estudiantes de literatura, 
La siguiente Bibliografía demuestra, por su extensión, el interés que Julio Cortázar ha provocado y sigue provocando. Se ha tratado de ofrecer una lista completa y correcta de libros y artículos de y sobre Cortázar aparecidos hasta la fecha. El motivo de la exclusión de algunos artículos se debe a la imposibilidad de comprobar personalmente la corrección de la cita, especialmente cuando en la mención original se omiten datos importantes, como su fecha o paginación. Por otra parte, el número especial en que se publica este trabajo de por sí lo hace incompleto en el mismo momento en que aparece. Queda entonces para el futuro la tarea de poner al día esta Bibliografía.

Quiero agradecer a la Universidad de Illinois que, a través de su Research Board y del Departamento de Español, me facilitó la valiosa ayuda de Eileen Zeitz en la investigación, y al Graduate College que me otorgó una "Summer Faculty Fellowship," sin la cual no podría haber completado este trabajo.

\section{Martha Paley de Francescato}

University of Illinois.

\section{Bibliografía de Julio Cortázar (1938-1973)}

\section{A. Libros de Cortázar}

Denis, Julio (seud.). Presencia. Buenos Aires: El Bibliófilo, 1938. [Poemas].

Cortázar, Julio. Los reyes. Buenos Aires: Gulab y Aldabahor, 1949. 76 pp. [Poema dramático].

—. Bestiario. Buenos Aires: Ed. Sudamericana, 1951. 165 pp. [Relatos].

-. Final del juego. México: Los Presentes, 1956. [9 relatos]. $2^{\text {a }}$ ed. aumentada. Buenos Aires: Ed. Sudamericana, 1964. 196 PP. [18 relatos].

- Las armas secretas. Buenos Aires: Ed. Sudamericana, 1959. 222 pP. [Relatos].

- Los premios. Buenos Aires: Ed. Sudamericana, 1960. 427 pp. [Novela]. 
- Historias de cronopios y de famas. Buenos Aires: Ed. Minotauro, 1962. 155 pp.

—. Rayuela. Buenos Aires: Ed. Sudamericana, 1963.635 pp. [Novela].

- Todos los fuegos el fuego. Buenos Aires: Ed. Sudamericana, 1966. 197 pp. [Relatos].

- La vuelta al día en ochenta mundos. México: Siglo XXI Editores, 1967. 214 pp. [Ensayos, relatos, poesía].

- Buenos Aires, Buenos Aires. Buenos Aires: Ed. Sudamericana, 1968. 224 pp. [Texto. Acompaña las fotografías de Alicia d'Amico y Sara Facio].

- 62. Modelo para armar. Buenos Aires: Ed. Sudamericana, 1968. 269 p. [Novela].

- Casa tomada. Buenos Aires: Ediciones Minotauro, 1969. Páginas sin numerar. [Edición en traducción al diseño gtáfico por Juan Fresán].

—. Ultimo round. México: Siglo XXI Editores, 1969. 220 PP. [Ensayos, relatos, poesía].

- Relatos. Buenos Aires: Ed. Sudamericana, 1970. 647 PP. [Contiene la totalidad de los relatos correspondientes a cuatro libros: Bestiario, Final del juego, Las armas secretas y Todos los fuegos el fuego, reordenados "con arreglo a afinidades que nada tienen que ver con el orden temporal de su publicación; los títulos de las tres partes $-R i$ tos, Juegos, Pasajes - apuntan oblicua y a veces irónicamente a esas líneas de fuerza"].

- Viaje alrededor de una mesa. Buenos Aires: Cuadernos de Rayuela, 1970. 62 pp. [Exposición de Cortázar en la mesa redonda sostenida en París en abril de 1970 sobre el tema "El intelectual y la política"].

- Pameos y meopas. Barcelona: OCNOS. Editorial Llibres de Sinera, 1971. 135 pp. [Obra que recoge poemas escritos entre 1951 y. 1958].

—. Prosa del observatorio. Barcelona: Editorial Lumen, 1972. 79 Pp. [Texto de Cortázar. Las fotografías son de los observatorios del sultán Jai Singh (Jaipur, Delhi, de 1968. Colaboró en ellas Antonio Gálvez].

- Libro de Manuel. Buenos Aires: Editorial Sudamericana, 1973. 386 pp. [Novela]. 
B. Reseñas; artículos, Notas, ensayos; cartas, poemas y relatos No COLECCIONADOS dE CORTízar.

Cortázar, Julio. "La urna griega en la poesia de John Keats." Revista de Estudios Clásicos, 2 (1946), 45-91.

- - Enrique Wernicke: El señor cisne. [Reseña]. Los anales de Buenos Aires, Núms. 20, 21, 22 (oct-nov-dic, 1947), 158.

-. "Notas sobre la novela contemporánea." Realidad, 3 (mar-abr, 1948), 240-46.

- "Muerte de Antonin Artaud." Sur, Núm. 163 (mayo, 1948), 80-82.

- Graham Greene: The Heart of the Matter, [Reseña]. Realidad, 5 (ene-feb, 1949), 107-12.

- Leopoldo Marechal: Adán Buenosayres. [Reseña]. Realidad, 5 (mar-abr, 1949), 232-38.

—. "Un cadáver viviente." Realidad, 5 (mayo-jun, 1949), 349-50. [Sobre el surrealismo].

- Françoss Porché: Baudelaire - Historid de un alma. [Reseña]. Sur, Núm. 176 (jun, 1949), 70-74.

—. "Irracionalismo y eficacia." Realidad, 6 (set-dic, 1949), 250-59. [Reflexiones sobre el capítulo "Existencialismo y nazismo". de $\mathrm{Va}$ loración literaria del existencialismo por Guillermo de Torre].

—. Octavio Paz: Libertad bajo palabra. [Reseña]. Sur, Núm. 182 (dic, 1949). 93-95.

-. Cyril Connolly: La tumba sin sosiego. [Reseña]. Sur, Núm. 184 (feb, 1950), 61-63.

-. "Situación de la novela." Cuadernos Americanos, 3, Núm. 4 jul-ag, 1950), 223-43.

-. Victoria Ocampo: Soledad sonora. [Reseña]. Sur, Núms. 192-9394 (oct-dic, 1950), 284-97.

—. "Los olvidados." [Reseña]. Surt, Núms. 209-10 (mar-ậar, 1952), 170-72.

—. "Gardel." Sut, Núm. 223 (jul-ag, 1953), 127-29.

-. Carlos Viola Soto: Periplo. [Reseña]. Buenos Aires Literaria, Núm. 15 (dic, 1953), 57.63-

—- "Para una poética." La Torre, Núm. 7 (jul-set, 1954), 121-38.

- Edgar Allan Poe: Obras en prosa. Traducción, Introducción y notas por Julio Cortázar. Ediciones de la Universidad de Puerto Rico. Madrid: Revista de Occidente, 1956. 2 volúmenes.

-.. "Algunos aspectos del cuento," Gasa de las Américas, Núms. 15-16 
1962-63), 3-14. [Reproducido parcialmente en El Escarabajo de Oro. Núm. 21 (dic, 1963), 13, 16-19.]

—. "Carta a J. Carnevale." Cero, Núms. 3-4 (mayo, 1965), 37.

- "Sobre las técnicas, el compromiso y el porvenir de la novela." El Escarabajo de Oro, Boletín Núm. 1 (1965), 3, [Entrevista con Luis Mario Schneider].

—. "Sobre Leopoldo Marechal." El Escarabajo de Oro, Núm. 30 (1966), 66-67, 74.

- "de Julio Cortázar a Francisco de la Moza." Revista de la Universidat de México, 21, Núm. 12 (ag, 1967), 31.

—. "Julio Cortázar al 'Che.'" La Estafeta Literaria, Núm. 383, 18 noviembre 1967, p. 9. [Poemas].

—. "Los testigos." Insula, Núm. 252 (nov, 1967), 16. [Relato].

—. "Carta a Fernández Retamar (I)." Primera Plana, Nứm. 281, 14 mayo 1968 , PP. $72-74$.

- "Carta a Fernández Retamar (II)." Primera Plana, Núm. 282, 21 mayo 1968, pp. 76-77.

Collazos, Oscar, Julio Cortázar, y Mario Vargas Llosa. Literatura en la revolución y revolución en la literatura. México: Siglo XXI Editores, 1970. 118 Pp. [Ensayos de polémica].

Cortázar, Julio. "720 Círculos." Revista Iberoamericana, 37, Núm. 74 (ene-mar, 1971), 13-15. [Poema].

- "Algunos aspectos del cuento." Cuadernos Hispanoamericanos, Núm. 255 (mar, 1971), 403-16.

-.. "Policrítica a la hora de los chacales." Los Libros, Núm. 20 (jun, 1971), 9-10. [Poema referente al "caso Padilla"].

—. "Respuesta a comentarios de David Viñas, en carta dirigida a Saúl Sosnowski." Hispamérica, Núm. 2 (dic, 1972), 55-58.

C. Traducciones de obras de Cortäzar

En demán:

Cortázar, Julio. Das besetzte Haus. Tr. Edith. Aron. Neuwied; Berlin: Luchterhand, 1963. 250 pp. [Casa tomada].

-. Gescbicbten der Cronopien und Famen. Tr. Wolfgang Promies. Neuwied; Berlin: Luchterhand, 1966. 1952 Pp. [Historias de crono. pios y de famas].

- Die Gewimner. Tr. Christa Wegen. Neuwied; Berlin: Luchterhand, 1966. Sonderausgabe, 1969. 468 Pp. [Los premios]. 
En checo:

Cortázar, Julio. Pronásledovatel. Tr. Kamil Uhlír. Praha: Odeon, 1966. 166 pp. [Bestiario; Final del juego; Las armas secretas (selec.)].

En francés:

Cortázar, Julio. Les gagnants. Tr. Laute Guille. Paris: A. Fayard, 1961. $384 \mathrm{pp}$. [Los premios].

$\longrightarrow$ Les armes sectètes. Tr Laure Guille. Paris: Gallimard, 1963. 227 PP. [Las armas secretas].

-. Marelle. Tr. Laure Guille et Françoise Roset. Paris: Gallimard, 1966. 597 PP. [Rayuela].

—_. Gîtes. Tr. Laure Guille-Bataillon. Paris: Gallimard, 1968. 255 Pp. [Las armas secretas (selec.); Bestiario (selec.); Findl del juego (selec.)].

—. Tous les feux, le feu. Tr. Laure Guille-Bataillon. Paris: Gallimard; 1970. $205 \mathrm{pp}$. [Todos los fuegos el fuego].

En bolandés:

Cortázar, Julio. De mierenmoordenaar. Tr. J. A. van Praag. Amsterdam: Van Ditmar, 1967. 142 pp. [Historias de cronopios y de famas].

- Brief aln een meisje in Parijs. Tr. J. A. van Praag. Amsterdam: Meulenhoff, 1969. 155 PP. [Bestiario (selec.); Final del juego (selec.)].

- Het kwijlen van de duivel. Tr. J. A. van Praag. Amsterdam: Meulenhoff; Bezige $\mathrm{By}$, 1969. 202 pp. [Las armas secretas; Todos los fuegos el fuego (selec.)].

En inglés:

Cortázar, Julio. The Winners. Tr. Elaine Kerrigan. New York: Pantheon, 1965. 374 pp. [Los premios].

- Hopscotch. Tr. Gregory Rabassa. New York: Pantheon, 1966. 564 pp. New York: New American Library, 1967. 448 PP. [Rayuela].

-. End of the Game and Other Stories. Tr. Paul Blackburn. New York: Pantheon, 1967. 277 pp. [Final del juego].

-Blow-up, and Otbers Stories. Tr. Paul Blackbum. New York: Collier, 1968. 248 pp. [Las armas secretas]. 
- Cronopios and Famas. Tr. Paul Blackburn. New York: Pantheon, 1969. 161 pp: [Historias de cronopios y de famas].

En italiano:

Cortázar, Julio. Le Armi Segrete. Tr. Cesco Vian. Milano: Rizzoli, 1963. 244 pp. [Las armas secretas].

—. Bestiario. . . Tr. Flaviarosa Nicoletti Rossini. Torino: Einaudi, 1965. 548 pp. [Bestiario; Las armas secretas; Final del juego].

- Storie di Cronopios e di Fama. Tr. Flaviarosa Nicoletti Rossini. Torino: Giulio Einaudi, 1971. 157 pp. [Historias de cronopios y de famas].

—. Il Gioco del Mondo. Tr. Flaviarosa Nicolletti. Torino: Einaudi, 1969. 523 pp. [Rayuela].

\section{En noruego:}

Cortázar, Julio. Seremonier. Tr. Kjell Risvik. Oslo: Gyldendal, 1970. 221 pp. [Bestiario; Las armas secretas; Final del juego; Todos los fuegos el fuego].

En polaco:

Cortázar, Julio. Tajemna brón. (Opowiadania). Tr. Zofia Chądzyñska. Warszawa: Państw. Instytut Wydawn, 1967. 226 pp. [Las armas secretar].

—. Gra w klasy. Tr. Zofia Chądzyńska. Warszawa: Czytelnik, 1968. 627 pp. [Rayuela].

- Dla wszystkich ten sam ogiéń. Tr. ZofiaChądzyńska. Warszawa: Szytelnik, 1969. 223 PP. [Todos los fuegos el fuego].

En portugués:

Cortázar, Julio. Blow-up e Outras Histórias. Tr. Maria M. Femandes Ferreira. Lisboa: Europa-América, 1968. 208 pp. [Las armas secretas].

O Jôgo da Amarelinba. Tr. Fernando de Castro Ferro. Rio de Janeiro: Civilização Brasileira, 1970. 521 PP. [Rayziela]. 
En rumano:

Cortázar, Julio. Sfî̀şitul jocului. Tr. Irina Ionescu şi Dumitru Tepeneag. București: Editura pentru literatură universală, 1969. 376 PP. [Las armas secretas; Historias de cronopios y de famas (selec.)].

En sueco:

Cortázar, Julio. Slut på leken. Noveller. Tr. Jan Sjögren. Stockholm: Bonnier, 1969. 231 pp. [Bestiario; Final del juego; Las armas secretas ].

D. Libros sobre Cortázar

Alascio Cortázar, Miguel. Viaje alrededor de una silla. Buenos Aires: Carpeta Editora, 1971.

Esta obra es una respuesta a Viaje alrededor de una mesa de Cortázar. En ella no se pretende enjuiciar la posición del escritor, "sino explicitar algunos de los disentimientos surgidos del análisis del citado opúsculo" (18). Sin embargo, un sentimiento muy negativo rige estas páginas; la crítica al escritor se agudiza: Viaje alrededor de una mesa, dice Alascio Cottázar, cae en el absurdo; su autor tiene una idea estrecha de la realidad y "plantea la creación literaria desde un ángulo irracional" (50). Además, su conducta es incoherente (57) y el escritor vive en "permanente desajuste" (58). Alascio Cortázar acepta que la obra literaria de Julio Cortázar es apasionante, pero enjuicia la posiciớn política del escritor.

Amícola, José. Sobre Corhtzar. Buenos Aires: Ed. Escuela, 1969.

Amícola se propone acercarse "a la obra literaria de Cortázar desde múltiples aspectos para que el lector tenga una idea de conjunto" (191), objetivo expresado por el crítico en la "Conclusión." Es un libro caó-" tico, en el que no se sigue un plan ordenado como lo demuestran en especial el capítulo VII, "Relación entre su vida y su obra" (141-44), cuatro páginas colocadas en ese lugar sin solución de continuidad con lo que antecede y lo que sigue, y el IX, "Análisis de algunos de sus per̂̀najes y obras," donde se procede arbitrariamente y sin explicación, en la selección. El capítulo VII, "Las clases sociales en su obra," no aporta 
nada significativo a la crítica literaria, y la "Conclusión," de sólo dos párrafos, es pobre. Este es otro buen ejemplo del afán de los críticos por escribir un libro sobre Cortázar sin miras a la calidad del producto. No hay bibliografía y las notas son escasas.

Aronne Amestoy, Lida, Cortázar: la novela mandala. Buenos Aires: Fernando García Cambeiro, 1972.

Este nuevo libro sobre una obra que todavía of rece material amplio para la crítica constituye un aporte en gran parte positivo e interesante. Está dividido en cinco capítulos (sin numeración), introducción, conclusión y una guía bibliográfica que desconoce (o pasa por alto) muchas obras críticas de importancia. De acuetdo a lo expresado por la autora, el enfoque de este libro es "por fuerza parcial y deja deliberadamente de lado la evaluación estética y el análisis literario" (13), lo cual no creemos que consiga hacer efectivamente, pues son aspectos integrales de algunos métodos de crítica literaria. El libro está en realidad "cortado" en dos: la primera parte que hace una comparación de Rayuela con Ulysses de Joyce está un poco separada del resto donde se examina la novela de Cortázar en detalle. Los mayores aciertos los encontramos en el capítulo titulado "Los caminos" que a su vez se subdivide en cinco partes, de las cuales la primera, "La literatura," es una de las más brillantes. El capitulo titulado "El encuentro" es también excelente. En general, sin embargo, da la impresión de una obra muy desigual. A pesar de que por momentos llega a descifrar, alumbrar ciertos aspectos, cae con frecuencia en la repetición de lo que ya ha sido enunciado por otros críticos anteriormente. La parte más débil es la conclusión, anticlimática en un estudio de esta naturaleza, al ofrecer la siguiente premisa: "Rayuela es una rayuela y su protagonista es el lector. No basta con saber leer, entonces. Hay que aprender a jugar", etc. (97). Como se ha expresado al principio, esta obra crítica es de interés y contiene algunos aportes valiosos que superan los defectos de organización y estructuración del material y de algunos de los juicios enunciados.

Escamilla Molina, Roberto. Julio Cortázar: visión de conjunto. México: Organización Editorial Novaro, 1970.

Como el "propio autor expresa, este trabajo, escrito con vacilación, sugiere y apunta, no atestigua. La actitud es de amor y devoción sin reservas. El primer capítulo analiza el cuento fantástico y lo metafísico 
en la novela porteña, y desde el capitulo II en adelante el autor se concentra en Cortázar y su obra. Libro de estructura débil y estilo deficiente, su aporte a la interpretación de la obra de Cortázar es mínimo. Solamente en las conclusiones podemos encontrar afirmaciones críticas de algún mérito, a pesar de estar introducidas por el siguiente enunciado: "La constante de mayor significación en la obra narrativa de Julio Cortázar se traduce en el rigor de su actitud moral" (176).

Filer, Malva E. Los mundos de Julio Cortázar. New York: Las Américas Publishing Company, 1970.

Esta obra no llega a desarrollar ningún tema en extenso y por lo tanto resulta demasiado fragmentada. Tiene cierta importancia como texto de referencia para análisis e interpretación de algún cuento en particular o algún tema en especial. Las acusaciones de índole política que se hacen al escritor en la última parte carecen de valor y no tienen cabida en una obra de crítica literaria. La bibliografía es útil y en general, se agregan algunos datos de interés a la crítica de Cortázar.

García Canclini, Néstor. Cortázar. Una antropologia poética. Buenos Aires: Ed. Nova, 1968.

Basándose en la premisa que el talento de Cortázar es predominantemente poéico, García Canclini analiza la obra de Cortázar en base a las imágenes poéticas que liberan la esencia de lo humano. Ve en la obra de Cortázar dos imágenes que son claves: la del laberinto y la de lo monstruoso. El libro está cuidadosamente estructurado y las observaciones son acertadas, a pesar de que falta una conclusión que resuma eficazmente lo que el crítico ha querido expresar a través de este análisis de algunos aspectos de la obra de Cortázar. La bibliografía es extensa, a pesar de padecer de ciertos defectos como omisiones de paginación, fechas de algunos artículos y la atribución errónea de la reseña sobre la Oda a la alegría y otros poemas de Eduardo González Lanuza a Cortázar, cuando el mencionado trabajo pertenece en realidad a Martínez Estrada.

Mac Adam, Alfred. El individuo y el otro. Critica a los cuentos de Julio Cortázar. Buenos Aires, New York: Ediciones La Libreria, 1971.

Mac Adam cree que conviene que la crítica repase toda la obra de Cortázar anterior a Rayuela, "porque allí están los elementos originales 
del autor" (13), creencia correcta que falla un tanto en la realización, especialmente en la parte central del libro donde se analizan los cuentos de Cortázar. Mac Adam lleva la comparación con Kafka a extremos inaceptables (ver en especial p. 71). Su interpretación en cuanto al humor de Cortázar es errónea, especialmente cuando habla del "humor negro" de "Carta a una señorita en París." En general, insiste en esta interpretación, como al referirse al "uso ligeramente humoristico de un tema que el autor toma seriamente" (101) hablando de "Axolotl," o del "momento semicómico" (136) en "La salud de los enfermos" cuando muere la madre. La estructura es deficiente: después de la "Conclu. sión" se agrega un Apéndice sobre Historias de cronopios y de famas. La mala calidad de la impresión contribuye a colocar este libro entre los más perecederos de la crítica sobre Cortázar.

Rein, Mercedes. Julio Cortázar: el escritor y sus máscaras. Montevideo: Ed. Diaco, 1969.

La intención de este trabajo es tratar de entender a Cortázar, "ante todo como artista, pero también como pensador que tiene algo que decir y lo hace a través del arte" (5-6). En efecto, Mercedes Rein lleva a cabo un análisis profundo y acertado de la obra de Cortázar, colocándolo primero dentro de la perspectiva histórica. Es de sumo interés el capítulo III, " $\mathrm{La}$ coordenada estética," en el que se analizan varios aspectos del escritor y en el que sobresale el apartado "El narrador y sus máscaras" que estudia la relación entre el narrador y el lector, y los métodos que usa el escritor para obtener el distanciamiento que caracteriza algunos de sus mejores cuentos. Los últimos dos capítulos plantean el problema de la posición ideológica en la literatura con objetividad. En general, esta obra constituye un aporte de alto interés a la crítica más reciente sobre Julio Cortázar.

Sola, Graciela de. Julio Cortazar y el hombre nuevo. Buenos Aires: Ed. Sudamericana, 1968.

Este libro es, sin duda, el mejor que se ha escrito hasta la fecha sobre Julio Cortázar. La intención ha sido "rastrear, desde los primetos escritos del autor, las líneas de fuerza que... van desplegándose con indudable constancia y particular sentido evolutivo en toda su obra" (8-9). Una de las características de Cortázar que Graciela de Sola intuye y desarrolla con gran acierto es su condición de "irrenunciable y profundo 
poeta" (15). El análisis de la obra de Cortázar es excelente y lúcido, y la cuidadosa estructura del libro contribuye a su valor. Esta es una obra de necesaria lectura para el que quiera comprender la obra- del escritor y sirve como base firme y excelente punto de partida de todo intento de crítica.

Sosnowski, Saúl. Julio Cortázar: Una búsqueda mítica. Buenos Aires: Ediciones Noé, 1973, No se ha podido consultar esta obra.

\section{E. Libros de conjunto sobre Cortízar.}

Cinco miradas sobre Cortázar. Buenos Aires: Ed. Tiempo Contemporáneo, Colección Números, 1968.

Las "cinco miradas" a que hace referencia el título están divididas en tres partes. La primera es una discusión sobre Rayuela, con un panel compuesto por Ana María Simo, José Lezama Lima y Roberto Fernández Retamar (7-82), y que constituye la parte más extensa del libro. La discusión tiene aspectos de interés y se desarrolla de manera espontánea. Los participantes presentan su punto de vista y sus opiniones; así, se logra establecer una conversación ágil que ofrece enfoques de valor sobre una de las obras más importantes del escritor. La segunda parte consiste en preguntas hechas a Cortázar por Mario Vargas Llosa, publicadas en el diario "Expreso" el 7 de febrero de 1965 (83-91). A pesar de la brevedad, es un trozo de valor, debido en gran parte a la calidad de las preguntas y comentarios al margen de Vargas Llosa y al interés de las respuestas que da Cortázar. $L a$ tercera parte es la ya conocida carta de Cortázar a Fernández Retamar del 10 de mayo de 1967 (93114), donde por primera vez Cortázar se definiera políticamente, y que desde entonces ha circulado por varias publicaciones.

Homenaje a Julio Cortázar. Helmy F. Giacomán, ed. Madrid: Anaya, 1972.

Contiene: Helmy F. Giacomán, "Prefacio," pp. 9-12; José Lezama Lima, "Cortázar y el comienzo de la otra novela," pp. 13-29; Marcelo Alberto Villanueva, "El salto hacia adelante o la razón de la sinrazón," pp. 31-80; Fernando Alegría, "Rayuela o el orden del caos", pp. 8194; Enrique Giordano, "Algunas aproximaciones a Rayuela, de Julio Cortázar, a través de la dinámica del juego," pp. 95-129; John G. Co- 
peland, "Las imágenes de Rayuela," pp. 131-49: Angela Dellepiane, "62. Modelo para armar: ¿Agresión, regresión o progresión?," PP. 151-80; Ana María Pucciarelli, "Notas sobre la búsqueda en la obra de Cortázar," Pp. 181-93; Malva E. Filer, "La búsqueda de autenticidad," pp. 195-206; Osvaldo López Chuhurra, ". . Sobre Julio Cortázar," pp. 207-34; José Blanco Amor, "Julio Cortázar," Pp. 235-60; Malva E. Filer, "Las transformaciones del yo," pp. 261-76; Antonio Pagés Larraya, "Cotidianeidad y fantasía en una obra de Cortázar," pp. 277-87; Alfred J. Mc Adam, "Los premios: Una tentativa de clasificación formal," pp. 289-96; Richard F. Allen, "Temas y técnicas del taller de Julio Cortázar," pp. 297-305; Flora H. Schiminovich, "Cortázar y el cuento en uno de sus cuentos," pp. 307-17; Germán D. Carrillo, "Emociones y fragmentaciones: técnicas cuentísticas de Julio Cortázar en "Todos los fuegos el fuego," PP. 319-28; Marta Morello-Frosch, "El personaje y.su doble en las ficciones de Cortázar," pp. 329-38); Joan Hartman, "La búsqueda de las figuras en algunos cuentos de Cortázar," PP. 339-50; "Wolfgang A. Luchting, "Todos los juegos el juego," Pp. 351-63; Martha Paley de Francescato, "Julio Cortázar y un modelo para armar ya armado," pp. 365-73; David Lagmanovich, "Estructura de un cuento de Julio Cortázar: "Todos los fuegos el fuego," pp. 375-87; Manuel Durán, "Julio Cortázar y su pequeño mundo de cronopios y famas," pp. 389-403; Roberto Hozven V., "Interpretación de 'El río.' cuento de Julio Cortázar," pp. 405-25; Saúl Sosnowski, "Conocimiento poético y aprehensión racional de la realidad. Un estudio de 'El perseguidor,' de Julio Cortázar," pp. 427-44; Luis Bocaz Q., "'Los reyes' o la irrespetuosidad ante lo real de Cortázar," Pp. 445-55; Antonio Pagés Larraya, 'Perspecticas de 'Axolotl,' cuento de Julio Cortázar," Pp. 457-80; Mary E. Davis, "Cortázar y Nabokov: La estética del éxtasis," Pp. 481-89;

Material recogido de libros y revistas, y monografías inéditas, componen este volumen de homenaje al escritor.

La vuelta a Cortázar en nueve ensayos. Recopilación y prólogo de Sara Vinocour de Tirri y Néstor Tirri. Buenos Aires: Carlos Pérez Editora, 1968.

Comprende: a) Cuentos: Noé Jitrik, "Notas sobre la Zona Sagrada y el mundo de los otros en Bestiario de Julio Cortázar," pp. 13-30 (1968); Manuel Durán, "Julio Cortázar y su pequeño mundo de cro nopios y famas," pp. $31-49$ (Revista Iberoamericana, ene-jun, 1965); 
Alain Bosquet, "Las realidades secretas de Julio Cortázar," pp. 51-54 (tr. de Le Monde de París, 3 août 1963); Alejandra Pizarnik, "Nota sobre un cuento de Julio Cortázar: 'El otro cielo," " pp. 55-62; b) Novelas: Antonio Pagés Larraya, "Los premios," Pp. 65-73 (pub. en Ficción, Núm. 33-34, set-dic, 1961); Graciela de Sola, "Rayuela: una invitación al viaje," Pp. 75-102; c) Todo Cortázar: Guillermo Ara, "Cortázar Cronopio" (Notas sobre La vuelta al día en ochenta mundos), PP. 105-18; Luis Gregorich, "Julio Cortázar y la posibilidad de la literatura," PP. 119-31; Néstor Tirri, "El perseguidor perseguido," pp. 133-61. Sara Vinocour de Tirri, "Referencias bibliográficas," Pp. 163-65; "Ediciones en lenguas extranjeras de las obras de Julio Cortázar," pp. 166-67.

A pesar de que algunos de los artículos recogidos en este volumen ya habian sido publicados con anterioridad, el libro es un aporte de valor a la crítica de Cortázar debido al indiscutible mérito de algunos trozos. Se destacan en especial los escritos de Jitrik, Durán, Bosquet, Pizarnik, de Sola, y Néstor Tirri. La selección bibliográfica no es de mayor importancia y contiene algunas erratas.

\section{F. Tesis doctorales}

Aronne de Amestoy, Lida. " 'Rayuela,' otra manera del compromiso literario," Mendoza, 1970.

Bates, Merritt W. "Dos autores, cuatro novelas." Universidad Nacional del Litoral, Rosario-Santa Fe-Argentina, 1968.

Brody, Robert. "Julio Cortázar in his Rayzela." Harward, 1971.

Chazelle, Marie-Christine. ' 'Omnibus,' un cuento de Julio Cortázar. (Edición anotada y estudio crítico)." Toulouse, 1969.

Childs, Tonya Lee. "Existentialism in the Works of Julio Cortázar." Texas, 1971.

Davis, Mary Eunice. "The Vision of Reality in Selected Novels by Sábato, Cortázar, and García Márquez." Kentucky, 1970.

Francescato, Martha Paley de. "El Bestiario de Julio Cortázar: enriquecimiento de un género." Illinois, Urbana-Champaign, 1970.

Garfield, Evelyn Picon. "La influencia del surrealismo francés en la obra de Julio Cortázar." Rutgers, 1972.

Holsten, Kenneth Alden. "The Metaphysical Search in the Novels of Julio Cortázar," California, San Diego, 1970.

Kerr, Lucille. "The Beast and the Double: A Study of the Short Stories of Julio Cortázar." Yale, 1972. 
Larisgoitia, Hernani. "Estudio analítico-interpretativo de los cuentos de Julio Cortázar." Wisconsin, 1971.

Mac Adam, Alfred John. "The Individual and the Other: A Study of the Prose Works of Julio Cortazar." Princeton, 1969.

$\mathrm{MaCoy}$, Katherine Wallis. "Theory of the Novel in Rayuela." Emory. 1970.

Martins, Teresinha Alves Pereira. "Julio Cortázar, Clarice Lispector e a Nova Narrativa Latinoamericana." New Mexico, 1971.

Muzika, H. M. "Rayuela, a Metaphysical Revolution." Liverpool, 1969.

Nelson, Kathleen G. "Julio Cortázar's Rayuela as an Existentialist Novel." Catholic University, 1971.

Osta, Winifred. "The Journey Pattern in Four Contemporary American Novels." Atizona, 1970.

Sosnowsky, Saúl "Julio Cortázar, una búsqueda mítica." Virginia, 1970. Spencer, Sharon D. "The Architectonic Novel: A Study of Structure in Modern Fiction Based Upon Works by Twenty-Three Experimental Novelists From Raymond Roussel to Carlos Fuentes." New York University, 1969.

G. ARtículos, NOTAS Y RESEÑas dE LAS OBRAS SOBRE CORTÁZAR

Alegría, Fernando. "Rayuela: o el orden del caos." Revista Iberoamericana, 35, Núm. 69 (set-dic, 1969), 459-72.

Allen, Richard F. "Los temas del tiempo y la muerte en Todos los fuegos el fuego de Julio Cortázar." Duquestre Hispantic Review, Núm. 3 (invierno, 1967), 35-50.

- "Temas y técnicas del taller de Julio Cortázar." XIII Congreso Internacional de Literatura Iberoamericana. Caracas: Organización del Bienestar Estudiantil, 1968, Pp. 289-96.

- Julio Cortázar: Todos los fuegos el fuego. [Reseña]. Books Abroad, 42, No. 1 (Winter, 1968), 80-81.

- "En busca de la novelística de Néstor Sánchez y Julio Cortázar." Cuadernos Hispanoamericanos, Núm. 237 (set, 1969), 711-25. [En realidad, es sobre Nosotros dos de Sánchez, pero muestra la influencia de Cortázar].

Alonso, J. M. "Cortázat, Borges and the Loss of Experience." Review 72 (Winter, 1972), pp. 14-17.

Alves Pereira, Teresinha. "Coincidencia de la técnica narrativa de Julio 
Cortázar y Clarice Lispector." Nueva Narrativa Hispanoamericana, 3, Nủm. 1 (ene, 1973), 103-11.

Amorós, Andrés. Julio Cortázar: 62. Modelo para armar. [Reseña]. Revista de Occidente, Núm. 73 (abr, 1969), 123-24.

Anderson Imbert, Enrique. Julio Cortázar: Final del juego. [Reseña]. Revista Iberoamericana, 23, Núm. 45 (ene-jul, 1958), 173-75.

Andreu, Jean L. "Todos los fuegos el fuego, les derniers contes de Julio Cortázar." Cahiers du Monde Hispanique et Luso-Brésilien (Caravelle), No. 8 (1967), 153-59. [La traducción de este artículo, "Cortázar cuentista," se publicó en Mundo Nuevo, Núm. 23 (mayo 1968), 87-90.].

- "Pour une lecture de Casa tomada de J. Cortázar." Cabiers du Monde Hispanique et Luso-Brésilient (Caravelle), No. 10 (1968), 49-60.

—. et Yves-René Fonquene. "Bestiario de Julio Cortázar: essai d'interprétation systématique." Cabiers du Monde Hispanique et LusoBrésilien (Caravelle), No. 11 (1968), 112-29.

Anónimo. Julio Cortázar: Cronopios and Famas. [Reseña]. Time, 13 June 1969, p. 105.

- Julio Cortázar: Ultimo Round. [Reseña]. La Nación, Supl. literario, 11 enero 1970, P. 5.

Anzoategui, Ignacio B. "El anti-Cortázar." Atlántida, Núm. 1234 (ene, 1970), 58-63. [Respuesta a las declaraciones de Cortázar a Life, es en realidad un violento ataque al escritor, a quien llama "peluquero de señoras," y donde se demuestra una total incomprensión de la obra del escritor y un marcado mal gusto, que le niegan al artículo todo valor crítico.].

Armani, Horacio. "Algunas vueltas en torno de Cortázar." La Nación, Supl. literario, 28 enero 1968, p. 4. [Sobre La vuelta al día en ochenta mundos].

Arrufat, Antón. Julio Cortázar: Cuentos. Selección y prólogo por Antón Arrufat. Cuba: Casa de las Américas, 1964, pp. vii-xvi.

—. "Las armas secretas de Julio Cortázar." Casa de las Américas, Núm. 26 (oct-nov., 1964).

Babín, María Teresa. "La antinovela en Hispanoamérica." Revista Hispánica Moderna, Núms. 3-4 (jul-oct, 1968), .523-32.

Barnatan, Marcos Ricardo. "Julio Cortázar, fantasma y escritor." Papeles de Son Armadans, 44, Núm. 132 (mar, 1967), 350-59.

—. "El retomo a Cortázar." Insula, Núm. 271 (jun, 1969), 13. 
Barrenechea, Ana María. "Rayuela, una búsqueda a partir de cero." Sur, Núm. 288 (may-jun, 1964), 69-73.

-. "La estructura de Rayuela, de Julio Cortázar." Litterae Hispanae et Lusitande. München: Max Hueber Varlag, 1968, pp. 69-84.

- "La estructura de Rayuela, de Julio Cortázar." Nueva Novela Latinoamericana 2. Buenos Aires: Editorial Paidós, 1972, Pp. 22247. [Artículo fechado noviembre 1968].

Barufaldi, Rogelio. "Soledad y vocación en la última narrativa argentina." Señales, Núm. 134 (ene-feb, 1962), 6-9).

Basso, Eleonora. "La música en la' obra de Cortázar." Revista Ibero. americana de Literatura, 2a. época, Núm. 2 (1970), 51-60.

Bataillon, Laure. "Sur deux tableaux." La Quinzaine littéraire, No. 132, 1er. au 15 janvier 1972, pp. 4-5. [Sobre 62. Modelo para armar].

Benasco, Rodolfo. "Explica a Julio Cortázar." Bibliograma, Núms. 35 36 (1967).

Benedetti, Mario. "Julio Cortázar, un narrador para lectores cómplices." Los Tiempos Modernos, Núm. 2 (abr, 1965), 16-19. [Reproducido en Letras del Continente Mestizo. Montevideo: Arca, 1967, pp. 85-103].

Bernu; Michele. "Essai d'interprétation d'un conte de Julio Cortázar: Contivuidad de los parques." Cabiers du Monde Hispanique et Luso-Brésilien (Caravelle), No. 13 (1969), 23-31.

Bersani, Leo. 62: A Model Kit. Tr. Gregory Rabassa. [Reseña]. The New York Times Book Review (26 November, 1972), pp. 7, 43.

Bishop, Tom. Julio Cortázar: Cronopios and Famos. Tr. Paul Blackburn. [Reseña]. Saturday Review, 27 September 1969, pp. 26-27.

Bjurström, C. G. "Det fantastiska i litteraturen; ett samtal med Julio Cortázar." Bonniers Litterära Magdsin, 38 (maj-juni, 1969), 33440.

- "Julio Cortázar. Mon fantastique." La Quinzaine littéraire, No. 100, 1er. au 31 août 1970, pp. 16-19.

Blanco Amor, José. "Julio Cortázar." Cuadernos Americanos, Núm. 5 (set-oct, 1968), 213-37.

Block de Behar, Lisa. Analisis de un lenguaje en crisis. Montevideo: Editorial Nuestra Tierra, 1969.

Bocaz Q., Luis. "LOS Reyes o la irrespetuosidad ante lo real de Cortázar." Atenea, 166, Núm. 419 (ene-mar, 1968), 47-55.

Boldori, Rosa. "Cortázar = una novelística nueva." Setecientosmonos, Núm. 7 (dic, 1965). 
—. "La irrealidad en la narrativa de Cortäzar." Boletin de Literaturas Hispánicas, Núm. 6 (1966), 13-27.

- "Sentido y trascendencia de la estructura de Rayuela." Boletin de Literaturas Hispánicas, Núm. 6 (i966), 59-69.

Bordelois, Ivonne. Julio Cortázar: Los premios. [Reseña]. Cusdermos del Congreso por la Libertad de la Cultura, Núm. 55 (1961), 87-88.

Borello, Rodolfo A. "Un conjunto de estudios sobre Cortázar y. su obra." La Gaceta, 20 de agosto 1967, p. 2.

—. "El último combate de Julio Cortázar." Cuadernos Hispanoamericanos, Núm. 374 (jul, 1970), '165-73. [Sobre Ultimo Round].

Bottone, Mireya. "Cortázar en el testimonio." Boletin de Literaturas bispánicas Núm. 6 (1966), 85-92.

—. "Bibliografía de Cortázar." Boletin de Literaturas Hispánicas, Núm. 6 (1966), 93-94.

—. "Bibliografía sobre Cortázar." Boletín de Literaturas Hispánicas, Núm. 6 (1966), 95-96.

Bryan, C. D. B. Julio Cortázar: Cronopios and Famas. Tr. Paul Blackburn. [Reseña]. The New York Times Book Review, 15 June 1969, p. 4 .

—. "The Deluxe Model." Review 72 (Winter, 1972), pp. 32-34. [Sobre 62: $A$ model Kit].

Cammozzi Barrios, Rolando. Julio Cortázar: Todos los fuegos el fuego. [Reseña]. Revista de Ocoidente, 2a. época, Núm. 55 (oct, 1967), 115-17.

Campos, Jorge. Julio Cottázar: Rayuela. [Reseña]. Insula, Núm. 250 (set, 1967), 11.

Carnevale, Jorge. "Cortäzar o el verdadero rostro." Cero, Núm. 1 (set, 1964), 29. [Sobre Final del juego].

—. "Cronopiaje." Cero, Núms. 3-4 (mayo, 1965), 36.

Carranza, José María. Julio Cortázar: 62. Modelo para armar. [Reseña]. Revista Iberodmericana, 35, Núm. 69 (set-dic, 1969), 557-59.

Castelar, Diana. "Final del juego: un libro de cuentos de Julio Cortázar." Clarin Supl. Jiteratio, 23 julio 1964, p. 3.

Castelli, Eugenio. "Cortázar, Rayuela y la antinovela." Moyano. Benedetto. Cortázar. Rosario, Santa Fe: Ed. Colmegna, 1968, PP. 5173.

Castillo, Abelardo L. "Las armas secretas: cuentos de Julio Cortázar." El grillo de papel, Núm. 2 (ene, 1960), 19-20.

Cócaro, Nicolás. "De Julio Denis, poeta, a Julio Cortázar." Las letras. 
y el destino argentino. Buenos Aires: Editorial Sopena, 1969, Pp. 107:10.

Coddou P., Marcelo. Julio Cortázar: Todos los fuegos el fuego. [Reseña]. Atenea, 153, Núm. 413 (jul-set, 1966), 244-48.

Coll, Edna. "Cortázar y su Rayuela." Atenea (Revista de la Univesidad de Puerto Rico en Mayagüez), Nueva Serie, Núm. 2 (jun, 1967), 9-17.

—_. "Aspectos cervantinos en Julio Cortázar." Revista Hisplánica Moderrsa, Nủms. 3-4 (jul-oct, 1968), 596-604.

Concha, Edmundo. Julio Cortázar: Todos los fuegos el fuego. [Reseña]. Anales de la Universidad de Chile, 124, Núm. 138 (1966), 249-50.

Conte, Rafael. "Doce proposiciones para un festival Cortázar." Cuzadernos Hispanoamericanos, Núm. 222 (jun, 1968), 590-601. [Sobre La vuelta al dia en ochenta mundos].

- "Julio Cortázar o la esperanza de la destrucción" en Lenguaje y violencia: Introducción a la narrativa bispanoamericana. Madrid: Al-Borak, 1962, pp. 133-156.

Copeland, John G. "Las imágenes de Rajuela." Revista lberoamericana, 33, Núm. 63 (ene-jul, 1967), 85-104.

Copouya, Emile. "The Winners by Julio Cortázar." Saturday Review, 27 March 1965.

Csép, Attila. "El papel de lo fantástico en las obras de Cortázar." Acta Litteraria Academtae Scientiarum Hungaricae, 11 (1969) 281-90.

Curutchet, Juan Carlos. "Apuntes para una lectura de Cortázar." Cuadernos Hispanoamericanos, Núm. 223 (jul, 1968), 233-38. [Sobre Todos los fuegos el fuego].

—. "Cortázar, Años de Aprendizaje." Cuadernos Hispanoamericanos, Núm. 255 (mar, 1971), 561-72.

- - "Cottázar: Descubrimiento de una realidad-otra." Cuadernos Hispanoamericanos, Núm. 256 (abr, 1971), 153-64.

—. "Cortázar: Metodología de la rebelión." Cuadernos Hispanoamericanos, Núm. 260 (fez, 1972), 330-37.

-. "Cortázar: La crítica de la razón utópica." Cuadernos Hispanoamericanos, Núm. 261 (mar, 1972), 495-79.

Charosky, Claudio B. "Cronología de un derrumbe: Cortázar y Fuentes." Alacrán Azul, Núm. 1 (1970), 74-76.

Dalton, Roque. "Histotia de cronopios y de famas." Casa de las Américas, Núms. 20-21 (dic, 1963), 64-65. 
D'Amico; Alicia y Sara Facio. "Los juegos de Julio Cortázar." La Nación, Supl. literario, 4 junio 1967.

Dellepiane, Angela B. "62. Modelo para armar: Agresión, regresión o progresión?" Nueva Narrativa Hispanoamericana, 1, Núm. 1 (ene, 1971), 49-72.

Devoto, Daniel. Julio Cortázar: Los Reyes. [Reseña]. Realidad, 6 (setdic, 1949), 319-21.

Díaz Ruiz, Ignacio. "Julio Cortázar: La golondrina enfurecida." Latinoamérica. Antario/Estudios Latinoamericanos, Núm. 4 (1971), 157-66.

Diego, Eliseo. Julio Cortázar: Todos los fuegos el fuego. [Reseña]. Casa de las Américas, 7, Núm. 41 (mar-abr, 1967), 122-25.

Durán, Manuel. "Julio Cortázar y su pequeño mundo de cronopios y famas." Revista Iberoamericana, 31, Núm. 59 (ene-jun, 1965), 33 46. [Reproducido en La vuelta a Cortázar en nueve ensayos].

Durand, José. "Julio Cortázar: los cuentos del gigante." Américas, 15 abr, 1963), 39.43.

"8 x 8 = Giglish." Time, 29 Aptil 1966, p. 112. [On Hopscotch].

Eloy Martínez, Tomás. "La Argentina que despierta lejos." Primera Pland, Núm. 103, 27 octubre 1964, PP. 36-40.

Eyzaguirre, Luis B. "'Rayuela,' 'Sobre héroes y tumbas' y 'El astillero': Búsqueda de la identidad individual en la novela hispanoamericana contemporánea." Nueva Narrativa Hispanoamericana, 2, Núm. 2 (set, 1972), 101-18.

Fernández, Henry. "From Cortázar to Antonioni: Study of an Adaption." Focus on Blow-Up. Roy Huss, ed. New Jersey: Prentice Hall, 1971, pp. 163-67.

Fernández Santos, F. "Julio Cortázar, cronopio universal." Indice, Núms. 221-23 (1967), 10-13.

Fèvre, Fermin B. Julio Cortázar: Final de juego. [Reseña]. Criterio, Núm. 1462 (oct, 1964), 796.

—_."Las figuras del zodíaco." Primera Plana, Núm. 173 (1966?), 67. [Reproducido en Atlas (June, 1966)].

- Julio Cortázar: 62. Modelo para armair. [Reseña.] Criterio, Núms. 1565-66 (feb., 1969), 108-09.

Figueroa Amaral, Esperanza, "Dos libros de Cortázar." Revista Iberocmericana, 35, Núm. 68 (mar-ag, 1969), 377-83. [Sobre Ceremonias y 62. Modelo para armar].

Filer de Tunkelang, Malva. "Las transformaciones del yo en la obra de 
Julio Cortázar." Cuadernos Hispanoamericanos, Núm. 242 (feb., 1970), 320-34.

Flogstad, Kjartan. "Det trivielle som metafysikk: Introduksjon til Julio Cortázar." Vinduet, 24 (1970), 188-93.

Flores, Margarita G. "Siete respuestas de Julio Cortázar." Revista de la. Universidad de México, 21, Núm. 7 (uar, 1967), 10-13.

Fombona, Julieta. "Rayzula." Revista Nacional de Cultura, Núm. 180 (abr/mayo, jun, 1967), 79-82.

Ford, Aníbal. "Los últimos cuentos de Cortázar." Mundo Nuevo, Núm. 5 (nov, 1966), 81-84. [Sobre Todos lo fuegos el fuego].

Foster, David W. Julio Cortázar: 62. Modelo para armar. [Reseña]. Books Abroad, 43, No. 3 (Summer, 1969), 382.

Francescato, Martha Paley de. "Julio Cortázar y un modelo para armar ya armado." Cuadernos Americanos, Núm. 3 (mayo-jun, 1969), 235.41 .

- Julio Cortázar: Ultimo Round. [Reseña]. Revista Iberoamericana, 36, Núm. 72 (jul-set, 1670), 532-34.

- Julio Cortázar: Casa tomada. Tr. al diseño gráfico por Juan Fresán. [Reseña]. Revista Iberoamericana, 36, Núm. 73 (oct-dic, 1270), $670-71$.

Friedmann, Florinda. La wuelta a Cortázar en nueve ensayos y Cinco miradas obre Cortábar. [Reseña]. Sur, Núm. 318 (mayo-jun, 1969), 95-97.

Fuentes, Carlos. "La nueva novela latinoamericana." La Cultura en México, Supl. de Siempre, Núm. 128, 29 de julio de 1964, pp. I-VII y XIV-XVII. [Sobre Vargas Llosa, Cortázar y Carpentier. "La caja de Pandora," sobre Cortázar, PP. VII y XIV-XV].

- "Rayuela: la novela como caja de Pandora." Mundo Nuevo, Núm. 9 (mar, 1967), 67-69. [Originalmente este artículo fue publicado en La Quinzaine littéraire].

Garavito, Julián. Julio Cortázar: Gîtes. Tr. Laure Guille-Bataillon. [Reseña]. Europe, No. 473 (set, 1968), 17-18.

Garcia Canclini, Néstor. "La inautenticidad y el absurdo en la narrativa de Cortázar." Revista de Filosofia, Núm. 16 (1966), 65-77.

- "Cortázar: el acceso a la casa del hombre." La Capital, 15 noviembre 1967. p. 2.

-. "El país de los cronopios." Primera Plana, Núm. 262, 2 enero 1968, Pp. 49-50. [Sobre La vielta al día en ochenta mundos].

Garfield, Evelyn Picon. "The Exquisite Cadaver of Surrealism." Review 72 (Winter, 1972), pp. 18-21. 
Ghiano, Juan Carlos. "Rayuela, una ambición 'antinovelística" " La Nación, Supl. literario, 20 octubre 1963, p. 5.

Gimferrer, Pedro. "Notas obre Julio Cortázar." Insula, Núm. 227 (act, 1965), 7.

Gómez, Carlos Alberto. "Literatura y antropología. 62. Modelo para armar por Julio Cortázar." La Nación, Supl. literario, 8 diciembre 1968 , p. 5.

González, Manuel Pedro. "Reparos a ciertos aspectos de Rayuela." Co: loquio sobre la nouela bispanoamericana. México: Tezontle, 1967, Pp. 68-81. [Entre otros juicios, González califica a Rayzela de "contrahechura," "pastiche," "una invitación al tedio y un eficaz antídoto contra el insomnio," lo cual da una pauta de la calidad del artículo y de la seriedad y objetividad del crítico].

González Bermejo, Ernesto. "Julio Cortázar. Una apuesta a lo imposible." Cosas de escritores. Montevideo: Biblioteca de Marcha, 1971, 91-136.

González Echeverría, Roberto. " "La Autopista del Sur' and the Secret Weapons of Julio Cortazar's Short Narrative." Studies in Short Fiction, 8, No. 1 (Winter, 1971), 130-40.

González Lanuza, Eduardo. Julio Cortázar: La vuelia al dia en ocbenta mundos. [Reseña]. Sur, Núm. 312 (mayo-jun, 1968), 87-89.

-. "Causalidad y casualidad a propósito de 62. Modelo para armar, de Julio Cortázar." Sur, Núm. 318 mayo-jun, 1969), 72-75.

Graciarena, Edmundo. "Primera Plana: La revista y sus armas secretas." Hoy en la Cultura, Núm. 17 (nov-dic, 1964), 12.

Grande, Félix. "Fragmento para un homenaje a Rayuela." Indice, Núms. 221-23 (1967), 22.

Gregorich, Luis. "Julio Cortázar y la posibilidad de la literatura." Cucadernos de Critica, Núm. 3 (ag, 1966), 38-47. [Reproducido en La vuelta a Cortazar en nueve ensayos].

Gregorio, María Isabel de. "Rayuela." Boletin de Literaturas Hispínicas, Núm. 6 (1966), 43-58.

Grossvogel, David I. "Blow-up. The Forms of an Esthetic Itinerary." Diacritics, 11, No. 3 (Fall, 1972), 49-54. [Lo mejor que se ha escrito hasta la fecha sobre el cuento de Cortázar "Las babas del diablo" y su influencia en la película de Antonioni].

Guasta, Eugenio. "Los argentinos a través de una novela de Cortázar." Criterio, Núm. 1513-14, 24 diciembre 1960, Pp. 918-21.

Guerrero Marthineitz, Hugo. "La vuelta a Julio Cortázar en 80 preguntas." Siete dias, Núm. 311 (30 abr-8 may, 1973), sin paginación, Guibert, Rita. "Un gran esscritor y su soleddad." Life en español (7 abs, 
1969), pp. 43-55. [Respuestas a una serie de preguntas formuladas por escrito por Rita Guibert en nombre de Life].

- Seven Voices. New York: Alfred A. Knopf, 1973. [Entrevistas con Borges, Cabrera Infante, Cortázar, García Márquez, Asturias, Nerada y Paz].

Gyurko, Lanin A. "Cyclic Time and Blood Sacrifice in Three Stories by Cortázar." Revista Hispánica Moderna, Núm. 4 (oct-dic, 1969), 341-62. [Sobre "Todos los fuegos el fuego," "La noche boca arriba" y "El ídolo de las cícladas"].

- "Man as Victim in Two Stories by Cortázar." Kentucky Romanzce Quarterly, 19, No. 3 (1972), 317-35. [Sobre "Las armas sectetas" e "Instrucciones para John Howell"].

Hamilton, Carlos D. "La novela actual de Hispanoamérica." Cuadernos Americanos, Núm. 2 (mar-abr, 1973), 223-51. [Sobre Cortázar, pp. 230-33].

Harss, Luis. "Cortázar o la cachetada metafísica." Mundo Nuevo, Núm. 7 (ene, 1967), 57-74. [El mismo artículo fue publicado en Los nuestros. Buenos Aires: Ed. Sudamericana, 1966, Pp. 252-300]. ."Cortázar en su taller." Indice, Núms. 221-23 (1967), 19-24.

Hartmann, Joan. "La búsqueda de las figuras en algunos cuentos de Cortázar." Revista Iberoamericana, 35, Ním. 69 (set-dic, 1969), $539-49$.

Heker, Liliana. "Rayzela de Julio Cortázar." El Heraldo Cultural, 15, 20 febrero 1966, p. 7.

Hozven V., Roberto. "Interpretación de 'El río,' cuento de Julio Cortázar." Atened, 170, Núms. 4 1-22 (jul-dic, 1968), 57-77.

Irby, James E. "Cortázar's Hopscotch and Other Games." Novel: A Forum on Fiction, 1, No. 1 (Fall, 1967), 64-70.

Juin, Hubert. "LesArmes Secrètes." Le Lettres Françaises (feb, 1964), 3.

Kalda, Alexandre. "Julio Cortázar et Monteforte Toledo." Arts, No. 922 (1963), 2.

Keene, Donald. "Moving Snapshots." The New York Times Book Review, 10 April 1966, p. 1.

Lafourcade, Enrique. "Julio Cortázar y el neo-culturanismo." Imagen, No. 83, (oct, 1970), 6-8.

Lagmanovich, David. "Rayuela, novela que no lo es pero no importa." La Gaceta, 29 marzo 1964.

—. "Un cuento de Julio Cortázar." La Gaceta (12 dic, 1971), 2" sec., p. 1. [Sobre "La isla a mediodía"]. 
_._. "Rasgos distintivos de algunos cuentos de Julio Cortázar." Hispamérica, 1, Núm. 1 (jul, 1972), 5-15. [Sobre "Torito"", "Bestiatio," "Axolotl," "Omnibus," "Después del almuerzo," "La noche boca arriba," "Las puertas del cielo," "Continuidad de los parques"].

Lancelotti, Mario A. Julio Cortázar: Final del juego. [Reseña]. Sur, Núm. 291 (nov-dic, 1964), 87-89.

Lezama Lima, José. "Cortázar y el comienzo de la otra novela." Casa de las Américas, Núm. 49 (1968), 51-62. [Reproducido bajo el título de "Para llegar a Julio Cortázar" en México en la Cultura, Supl. de Novedades, Núm. 348 (oct., 1968), ii-vii].

Liberman, Anoldo. "Rayuela, por Julio Cortázar." Comentario, Núm. 38 (1964), 95-96.

Lockhart, Washington. "El esperanzado desbarajuste de Cortázar." Mar* cha, 15 setiembre 1967, p. 29.

López Chuhurra, Osvaldo. "Sobre Julio Cortázar." Cuadernos Hispanoamericanos, Núm. 211 (jul, 1967), 5-30.

López-Delpecho, Luis. "C. de Carroll y de Cortázar." Cuademos Hispanoamericanos, Núm. 228 (dic, 1968), 663-74.

López-Morales, Eduardo E. "El libro de los Julios." Casa de las Américas, Núm. 50 (1968), 172-78.

Loveluck, Juan. "Aproximación a Rayuela." Revista Iberoamericana, 34, Núm. 65 (ene-abr, 1968), 83-93.

Luchting, Wolfgang A. "Todos los juegos el juego." Mundo Nuevo, Núm. 35 (mayo, 1969), 29-35.

Llopis, Rogelio. "Prologando a Cortázar." Pueblo y Cultura, Núm. 30 (dic, 1964), 58-59.

Mac Adam, Alfred J. "'Los premios': Una tentativa de clasificación formal." XIII Congreso Internacional de Literatzra Iberoamericana. Caracas: Organización de Bienestar Estudiantil, 1968, pp. 101-08. [Posteriormente a su comunicación, presentada en la reunión en agosto de 1967, apareció en Mundo Nuevo, Núm. 18 (dic, 1967), 38, bajo el título "Cortázar novelista"].

- "El individuo y el otro." Revista Iberoamericana de Literatura, 2" épaca, Núm. 2 (1970), 3-16. [Sobre los ensayos críticos de Cortázat y especialmente sobre Los reyes].

- "La simultaneidad en las novelas de Cortázar." Revista Iberoamericana, 37, Núms. 76-77 (ju!-dic, 1971), 667-76.

—. "Cortázar on Cortázar: A Literary Chronology." Review 72 (Winter, 1972), pp. 35-41. 
Madrigal, Luis Iñigo. Julio Cortázar: Los premios. [Reseña]. Anales de la Universidad de Cbile, Núm. 123 (3er. ttimestre, 1961), 212-14.

Mastrángelo, Carlos. " "Las babas del dỉablo', Una Nueva Era Cuentística Argentina." El Contemporáneo, Núm. 5 (ag, 1969), 9.

—. "Usted, yo, los cuentos de Julio Cortázar y su autor." Revista de la Universidad Nacional de Córdoba, No. 10 (1969), 1085-1137.

Matilla Rivas, Alfredo. Julio Cortázar: La vuelta al día en ochenta mundos. [Reseña]. La Torre, Núm. 60 (1968), 301-05.

Micha, René. "Le Je et l'Autre chez Julio Cortázar." La Nouvelle Revue Françatise, No. 140, ler août 1964, pp. 314-22.

Miguel, André. Julio Cortázar: Les Armes Secrètes. [Reseña]. La Noutvelle Revue Française, No. 131, 1er novembre 1963, pp. 916-17.

Miguel, María Ester de. Julio Cortázar: Las armas secretas. [Reseña]. Señales, Núm. 114 (oct, 1959), 17-18.

Mirande, Nelly Donni de. "Notas sobre la lengua de Cortázar." Boletín de Literaturas Hispánicas, Núm. 6 (1966), 71-83.

Monsiváis, Carlos. "Instructivo para edificar un intersticio que permita encontrarle alguna utilidad a la nota bibliográfica o ¿qué pasaría, Eduviges, si todos los cronopios se llamaran Julio?" La Cultutra en México, Supl. de Siempre, Núm. 306, 27 diciembre 1967, v. [Sobre La vuelta al dia en ocbenta mundos].

—. "Bienvenidos al universo Cortázar." Revista de la Universidad de México, 22, Núm. 9 (mayo, 1968), 1-10.

Montesinos, Jaime A. "Contra la nada que acecha: Julio Cortázar." Cuadernos Americanos, 178 (set-oct, 1971), 237-43.

Morello-Frosch, Marta. "El personaje y su Doble en las Ficciones de Cortázar." Revista lberoamericana, 34, Núm. 66 (jul-dic, 1968), 323-30.

—. "Julio Cortázar: From Beasts to Bolts." Books Abroad, 44, No. 1 (Winter, 1970), 22-25.

Muñoz Martínez, Silveiro. "Otra mirada sobre Rayuela." Revista Iberoamericana de Literatura, 2" época, Núm. 2 (1970), 29-49.

Murena, H. A. Julio Cortảzar: Rayzela. [Reseña]. Cuadernos, Núm. 79 (dic, 1963), 85-86.

Musselwhite, David. "'El Perseguidor,' un modelo para desarmar," Nuevos Aires, Núm. 8 (ag/set/oct, 1972), 23-36.

Neruda, Pablo. "Con Cortázar y con Arguedas." El Universal (Caracas), 17 agosto 1969, p. 23.

"Novela espesa con sabias reminiscencias." Primerct Planza, Núm. 42, 27 agosto 1963, p. 34. [Sobre Rayuela]. 
Novoa, Leopoldo. "Cortázar o cómo venir al Sena permaneciendo en el Plata." Marcha, 9 julio 1965, p. 30.

Núñez, Antonio. Julio Cortázar: Ceremonias. [Reseña]. Cuaderizos Hispanoamericanos, Núm. 235 (jul, 1969), 234-36. [Título reúne dos libros de cuentos publicados con anterioridad: Final del juego y Las armas secretas].

Ojeda, Jorge Arturo. "Cronopios y. Famas." El Gallo Ilustrado, Supl. dominical de El Día, Núm. 287, 24 diciembre 1967.

Olaso, Ezequiel de. "El pastor de monstruos." La Nación, Supl. literario, 24 mayo 1964, p. 4.

Onega, Gladys S. "Cortázar: personajes y misterio." Setecientosmonos, Núm. 7 (dic, 1965).

—_. "Los premios." Boletin de Literaturas Hispanicas. Núm. 6 (1966), 29-41.

Orphée, Elvira. Julio Cortázar: Las armas secretas. [Reseña]. Sur, Nủm. 265 (jul-ag, 1960), 51-54.

Ortega, Julio. "Julio Cortázar/Rayuela." La contemplación y la fiesta. Ensayos sobre la novela latinoamericand. Lima: Ed. Universitaria, 1968, pp. 29-43.

Ortiz Orlando. "Un átomo de Rayuela." La Cultura en México, Supl. de Siempre, Núm. 825, 16 abril 1969, pp. vi-viii, [Sobre 62. Modelo para armar].

Oviedo, José Miguel. "Cortázar para recortar y pegar." La Cultura en México, Supl. de Siempre, Núm. 825, 16 abril 1969, pp. vi-viii. [Sobre 62. Modelo para armar].

Pagés Larraya, Antonio. Julio Cortázar: Los premios. [Reseña]. Ficción, Núms. 33-34 (set-dic, 1961), 165-69.

_. "Cotidianidad y fantasía en una obra de Cortäzar." Cuadernos Hispanoamericanos, Núm. 231 (mar, 1969), .694-703. [Sobre Historias de cronopios $y$ de famas].

_. "Perspectivas de 'Axolotl,' cuento de Julio Cortázar." Nueva Narrativa Hispantoamericana, 2, Núm. 2 (set, 1972), 7-24.

Perrone, Alberto M. "Julio Cortázar cuenta su vida y su obra." $A B C$ de las Américas, Núm. 34 (2-8 jun, 1973), 50-53.

Pichón Riviere, Marcelo. "Cortázar: De Rayuela al Libro de Manuel." Panorama, Núm. 313 (26 abr-2 may, 1973), 60-64.

—. "Julio Cortázar, hoy." Boletin de Literaturas Hispánicas, Núm. 6 (1966), 5-11. [Aparecido luego en su libro Estudios de literatura argentina. Buenos Aires: Ed. Galerna, 1969, pp. 157-72]. 
Rama, Angel. "Julio Cortázat: una novela distinta en el Plata." Marcha, 17 marzo 1961, p. 22.

Rambures, Jean-Louis de. "Cortázar, le troisième grand de la littérature sud-américaine." Réalité (fev, 1968), 88-91.

Rein, Mercedes. "A propósito de 'Las babas del diablo." Revista Iberoamericana de Literatura, $2^{\text {a }}$ época, Núm. 2 (1970), 17-28.

Revol, E. L. "La vuelta a Cortázar en 80 julios." Letras del Ectador, Núm. 137 (mayo-jun, 1968), 20,23.

—. "La tradición fantástica en la literatura argentina." Revista de Estudios Hispánicos, 2, Nủm. 2 (nov, 1968), 205-28. [Habla de Cortázar en extenso].

-. "Arena en los ojos." La Nación, Supl. literario (6 mayo, 1973), p. 6. [Reseña de Libro de Manuel].

Rial, José Antonio. "Reparos a Cortázar." El Universal (Caracas), 11 mayo 1969, p. 47.

Ríos, Roberto E. "Rayuela, de Julio Cortázar. Argentino. Primera edición, 1963." La novela y el bombre bispanoamericano. Buenos Aires: Ed. La Aurora, 1969, Pp. 103-16.

Rodríguez Monegal, Emir. "La nueva novela de Latinoamérica. La pluma busca otros horizontes." Life en español, 25, Núm. 6, 15 marzo 1965, PP. 57, 59.

—. "Erudición cortaziana." Mundo Nuevo, Núm. 8 (feb, 1967), 90-91.

-. "Los nuevos novelistas." XIII Congreso Internacional de Literatura Iberoamericana. Caracas: Organización de Bienestar Estudiantil, 1968, pP. 33-42.

—. "Le Fantôme de Lautréamont." Review 72 (Winter, 1972), PP. 26-31.

Rohon, Guy. Julio Cortázar: Marelle. [Reseña]. La Nouvelle Revue Française, No. 173, 1er août 1967, pp. 1113-15.

Rojas Guardia, Pablo. Julio Cortázar: La vuelta al dia en ocbenta mundos. [Reseña]. Revista Nacional de Cultura, Núm. 189 (jul-ag-set, 1969), 110-12.

-. La realidad mágica: Ensayos de Aproximación Literaria. Caracas: Monte Avila, 1969, Pp. 29-33.

Saez, Jorge Alberto. "Alrededor del hombre en ochenta mundos y tres julios." Sur, Núm. 311 (mar-abr., 1968), 87-90.

Salazar Bonday, Sebastián. Julio Cortázar: Bestiario. [Reseña]. Sur, Núm. 201 (jul, 1957), 109-10. 
Sanhueza, Ana María. "Caracterización de los narradores de Rayuela." Revista Cbilena de Literatura, Núm. 1 (otoño, 1970), 43-57.

Saporta, Marc. "Un fantôme d'Edgar Poe revu par Freud." L'Express, No. 887, 8-14 juillet 1968, p. 45. [Sobre Gîtes].

Sarduy, Severo. "Del Ying al Yang (Sobre Sade, Bataille, Marmori, Cortázar y. Elizondo)." Mundo Nuevo, Núm. 13 (jul, 1967), 4-13. [Reproducido en Escrito sobre th cuerpo. Enssayos de critica. Buenos Aires: Ed. Sudamericana, 1969, pp. 9-30.]

-. "Anamorphoses." La Quinzaine littéraire, No. 50, ler au 15 mai 1968, p. 5. [Sobre Gîtes].

Sasturain, Juan. Libro de Monuel. [Reseña]. La Opinión (10 may, 1973), p. 18.

Schiavo, Leda y Graciela Reyes. "'Modelo' y armado en una novela de Cortázar." Filologia, 15 (1971), 241-50.

Schmucler, Héctor. "Rayuela: juicio a la literatura." Pasado y Preserte, Núm. 9 (abr-set, 1965), 29-45.

-.. "Cortázar, escritor antiliterario." La Gaceta, 29 mayo 1966, p. 2.

-. "Notas para una lectura de Cortázar." Los libros, Núm. 2 (ag, 1969), 11. [Sobre 62. Modelo para armar].

Schneider, Luis Mario. "Entrevista a Julio Cortázar." Revista de la Universidad de México, 17, Núm. 8 (abr, 1963), 24-25.

Serra, Edelweis. "Comentario a Julio Cortázar a propósito de Final de juego." Señales, Núm. 147 (40. trimestre, 1964), 1-4.

Siracusa, Isabel. "Informe sobre la literatura argentina en 1969: Panorama de la critica." Nueva Critica, 1 (ene-abr, 1971), 62-133. [Sobre Cortázar, Pp. 78-83].

Skameta, Antonio. "Trampas al perseguidor." Mapocbo, Núm. 20 (verano, 1970), 33-44.

"Snapshots of Blow-up." Review 72 (Winter, 1972), Pp. 22-25. [Fragmentos de artículos: 1) Charles Thomas Samuels, "Sorting Things out in the Blow-up," Focus on Blow-Up, 1971); 2) Stanley Kauffmann, "The invisible immanence of evil," Foctus on Blow-Up; 3) Rita Guibert, "Cortázar defends Antonioni," Seven Voices].

S[oares], N. J. "Las tribulaciones de Cortázar." Primera Plana, Núm. 409, 10. diciembre 1970, p. 45. [Sobre Viaje alrededor de una mesa].

Sola, Graciela de. "Las galerías secretas de Julio Cortázar." Señales, Núm. 154 (3er trimestre, 1966), 3-6.

Soriano, Osvaldo. "Reportaje a Julio Cortázar." La Opinión (11 mar, 1973), PP. 18-20. 
Sosnowski, Saúl. "La intuición de la muerte en Las armas secretas de Julio Cortázar." Hispania, 52 (1969), 846-51.

- "El compromiso revolucionario de Cortázar: un nuevo 'round."' Nueva Narrativa Hispanoamericana, 2, Núm. 1 (ene, 1972), 211-13. [Sobre Viaje alrededor de una mesa].

Speratti Piñero, Emma Susana. "Ia literatura fantástica en las âlimas generaciones argentinas." La literatura fantástica en Argentina. México: Imprenta Universitaria, 1957, pp. 73-94. [Libro escrito en colaboración con Ana María Barrenechea].

Ullán, José Miguel. "Dos cronopios." Indice, Núms. 221-23 (1967), 18.

Uriarte, Fernando. "Julio Cortázar, novelista de Buenos Aires." Mapocbo, 14, Núms. 2-3 (1966), 57-67.

Uribe, Basilio. Julio Cortázar: Rayuela. [Reseña]. Criterio, Núm. 1452, 28 mayo 1964, p. 397.

Urondo, Francisco. "El escritor y sus armas politicas." Panorama, 24 noviembre 1970, pp. 40-50. [Entrevista a Cortázar en Buenos Aires].

Valbuena Briones, A. "Una cala en el Realismo Mágico." Cuadernos Americanos, Núm. 5 (set-oct, 1969), 233-41. [P. 237 en adelante sobro Cortázar].

Varela, Blanca. "Uno o muchos libros." Revista Pertiana de Cultura, Núm. 3 (oct, 1964), 130-34.

Vargas Llosa, Mario. "Entrevista a Julio Cortázar." Comentarios Bibliograficos Americanos, 3, Núm. 11 (ene-mar, 1971), 17-19.

Vázquez Amaral, José. "Julio Cortázar's Hopscotch and Argentinian Spiritual Alienation." The Contemporary Latin American Narrative. New York: 1970, pp. 157-65.

"Las verdades de Marechal." Análisis. Núm. 393, 25 setiembre 1968, PP. 50-54. [Entrevista; hay algunos juicios sobre Cortázar].

"Un viaje imaginario a través de un talento creador." Clarin, Supl. literario, 15 febrero 1968 , p. 5.

Viñas, David. "Después de Cortázar: historia y privatización." Cuadernos Hispanoamericanos, Núm. 234 (jun, 1969), 734-39. [Incidencia de Cortázar sobre la nueva generación de narradores argentinos: 1. Ricardo Piglia: Jaulario - 2a ed.: La invasion; 2. Aníbal Ford: Sumbosa; 3. Germán Garcia: Nanina; 4. Néstor Sánchez: Nosotros dos; 5. Ricardo Frete: Dos parientes; 6. Manuel Puig: La traición de Rita Haywortb].

—. "Cortázar y la fundación mitológila de París" y "Un viaje contra- 
dictorio: de Los premios a Rayuela" En De Sarmiento a Cortázadr: Buenos Aires: Ediciones Siglo Veinte, 1971, pp. 122-132 y 199-202. _. "Cortázar y la fundación mitológica de París." Nuevos Aires, 1, Núm. 3 (dicene-feb, 1971), 27-34.

Vittori, José Luis. "La voluntad de realismo." Colmegna (1963), 17-18. [Sobre Los premios].

"The Winners by Julio Cortázar." Time, 9 April 1965, p. 112.

Wood, Don E. "Surrealistic Transformation of Reality in Cortázar's Bestiario." Romance Notes, 13, No. 2 (Winter, 1971), 239-42.

Yahni, Roberto. "Los conciertos de Cortázar." Sur, Nüm. 319 (jul-ag, 1969), 56.59.

Yrache, Luis. "62. Modelo para armar." Papeles de Son Armadans, 57, Núm. 170 (mayo, 1970), 205-16.

Urbana, 15 de junio de 1973. 